\title{
Calculation of the intracellular elastic modulus based on an atomic force microscope micro-cutting system
}

\author{
YU Miao ${ }^{1}$, WANG JingHe ${ }^{1,2}$, WANG HongXiang ${ }^{2}$, LIU Li $^{2,3^{*}}$, YAN YongDa ${ }^{2}$, ZHANG JunJie ${ }^{2}$, \\ LIANG YingChun ${ }^{2} \&$ DONG Shen ${ }^{2}$ \\ ${ }^{1}$ State Key Laboratory of Robotics and System, Harbin Institute of Technology, Harbin 150001, China; \\ ${ }^{2}$ Center for Precision Engineering, Harbin Institute of Technology, Harbin 150001, China; \\ ${ }^{3}$ The Fourth Department of Neurosurgery, the First Affiliated Hospital of Harbin Medical University, Harbin 150001, China
}

Received April 29, 2011; accepted August 25, 2011; published online March 23, 2012

\begin{abstract}
Better understanding of variations in the mechanical properties of cancer cells could help to provide novel solutions for the diagnosis, prevention, and treatment of cancers. We therefore developed a calculation model of the intracellular elastic modulus based on the contact pressure between the silicon tip of an atomic force microscope and the target cells, and cutting depth. Ovarian cells (UACC-1598) and colon cancer cells (NCI-H716) were cut into sequential layers using an atomic force microscope silicon tip. The cutting area on the cells was $8 \mu \mathrm{m} \times 8 \mu \mathrm{m}$, and the loading force acting on the cells was increased from 17.523 to $32.126 \mu \mathrm{N}$. The elastic modulus distribution was measured after each cutting process. There were significant differences in contact pressure and cutting depth between different cells under the same loading force, which could be attributed to differences in their intrinsic structures and mechanical properties. The differences between the average elastic modulus and surface elastic modulus for UACC-1598 and NCI-H716 cells were $0.288 \pm 0.08 \mathrm{kPa}$ and $0.376 \pm 0.16 \mathrm{kPa}$, respectively. These results demonstrate that this micro-cutting method can be used to measure intracellular mechanical properties, which could in turn provide a more accurate experimental basis for the development of novel methods for the diagnosis and treatment of various diseases.
\end{abstract}

atomic force microscope, micro-cutting, intracellular elastic modulus

Citation: Yu M, Wang J H, Wang H X, et al. Calculation of the intracellular elastic modulus based on an atomic force microscope micro-cutting system. Chin Sci Bull, 2012, 57: 1868-1872, doi: 10.1007/s11434-012-5053-y

The cytoskeleton has a major effect on the responsiveness of cells to external stimuli [1-5] and is composed of a variety of protein filaments of different sizes and degrees of stiffness [6-10]. Cells can be cut into sequential layers using an atomic force microscope (AFM) tip, which allows the elastic modulus of the cytoskeleton and the organelles to be determined. The sequential distribution of the intracellular elastic modulus can thus also be plotted, allowing an overall understanding of the mechanical properties of the cells to be determined. This in turn can provide a more detailed experimental basis for studying cytopathology and disease processes. Investigations into intracellular mechanical properties are therefore crucial.

*Corresponding author (email: h_e2000@ hotmail.com)
In this study, cells were cut into sequential layers using an AFM-based nano-cutting system, and the contact properties between the AFM silicon tip and the cell were analyzed to measure the distribution of the intracellular elastic modulus. In addition, the cell morphology after each cutting process was also examined.

\section{Materials and methods}

\subsection{Sample preparation}

Ovarian cells (UACC-1598) and colon cancer cells (NCIH716) were treated with $10 \%$ fetal calf serum (volume fraction) and double-resistance (penicillin, streptomycin, final concentration $200 \mathrm{U} / \mathrm{mL}$ ) RPMI 1640 medium, and cultured 
in 12-well plates. Cells were grown on 1-cm diameter coverslips at $37^{\circ} \mathrm{C}$ under $5 \% \mathrm{CO}_{2}$. Well-grown cells were selected for the experiments. Cells were fixed with $5 \%$ glutaraldehyde for $15 \mathrm{~min}$ at room temperature.

\subsection{Instrument}

A Dimension 3100 AFM (Veeco, USA) was used. Silicon cantilevers with a nominal spring constant of $30 \mathrm{~N} / \mathrm{m}$ (Microlevers, MLCT-AUHW, Veeco, USA) were used for cutting.

\subsection{Experimental procedure}

The cell sample was fixed on the AFM scanning stage. The scanning parameters included a scan range of $30 \mu \mathrm{m} \times 30 \mu \mathrm{m}$, scan rate of $1 \mathrm{~Hz}$, an integral gain of $2 \mathrm{~V}$, and a proportion gain of $4 \mathrm{~V}$. After initialization, the tip approached the sample surface to penetrate the sample, accompanied by vertical loads imposed on the cells. The lateral edge of the tri-cone tip cut the sample in accord with the scanning motion of the tip. The cutting area was $8 \mu \mathrm{m} \times 8 \mu \mathrm{m}$. The indentation force curve on the processed region was detected, and the indentation lattice is shown in Figure 1.

\section{Results}

\subsection{Calculation of cutting depth and Hertzian contact stress between the silicon tip and the cell}

The AFM silicon tip was tri-cone shaped, with a spherical cap at the end of the tip [11]. Hertzian theory suggests that the contact between the hard sphere and the plane is assumed to be an elastic contact. To calculate the average stress of samples at different loads, the silicon tip was assumed to be spherical and the cell was considered to be a flat surface. The depth of penetration of the silicon tip into the cell surface can thus be expressed as

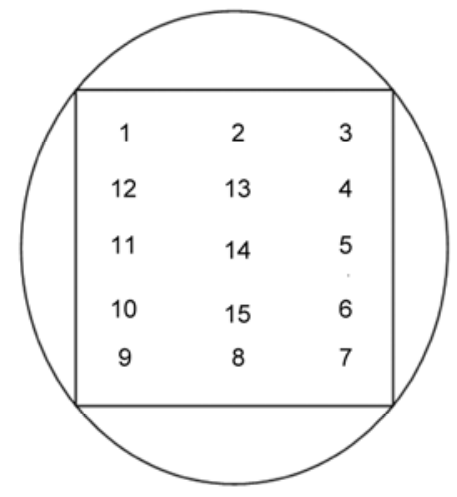

$$
\delta=\frac{2 K^{\prime}(k)}{\pi \lambda}\left[\frac{9 R}{16}\left(\frac{1-v_{\mathrm{Si}}^{2}}{E_{\mathrm{Si}}}+\frac{1-v_{\text {cell }}^{2}}{E_{\text {cell }}}\right)^{2} W^{2}\right]^{\frac{1}{3}},
$$

where $W$ is the exerted vertical loading force. $\lambda$ is a constant, and $\lambda=1$ when there is contact between the sphere and the plane. The radius, Young's modulus and Poisson's ratio of the silicon tip are $R(10 \mathrm{~nm}), E_{\mathrm{Si}}$ and $v_{\mathrm{Si}}$, respectively. The Young's modulus and Poisson's ratio of the cells are $E_{\text {cell }}$ and $v_{\text {cell }} \cdot 2 K^{\prime}(k) / \pi \lambda=1$, when there is contact between the sphere and the plane.

Once cutting began, the tri-cone part of the silicon tip became the effective working part as the interaction between the silicon tip and the cells increased. The contact area $A$ between the silicon tip and the cell is expressed as

$$
A=3 \sqrt{3}\left[\frac{3 R W}{4}\left(\frac{1-v_{\mathrm{Si}}^{2}}{E_{\mathrm{Si}}}+\frac{1-v_{\text {cell }}^{2}}{E_{\text {cell }}}\right)\right]^{\frac{1}{3}} .
$$

The Young's modulus and Poisson's ratio of the AFM tip were $190 \mathrm{GPa}$ and 0.28 , respectively. The surface elastic modulus of ovarian cells (UACC-1598) $E_{\text {cell1 }}$ was $4.35 \mathrm{kPa}$, and the Poisson's ratio $v_{\text {cell1 }}$ was 0.3 . The surface elastic modulus $E_{\text {cell2 }}$ and the Poisson's ratio $v_{\text {cell2 }}$ of colon cancer cells (NCI-H716) were $6.37 \mathrm{kPa}$ and 0.3 , respectively.

The side of the pyramidal edge of the tip acted as the cutting edge, and the maximum contact pressure between the tip and the cells is thus expressed as

$$
P_{\max }=\frac{3 W}{2 A}=\left[\frac{18 \sqrt{3} R}{W^{2}}\left(\frac{1-v_{\mathrm{Si}}^{2}}{E_{\mathrm{Si}}}+\frac{1-v_{\text {cell }}^{2}}{E_{\text {cell }}}\right)\right]^{-\frac{1}{3}} .
$$

The maximum contact pressure $P_{\max }$ and cutting depth $\delta$ of the cells under different loading forces are shown in Table 1.

As shown in Table 1, there were significant differences in contact pressure and cutting depth at the cell surface be-

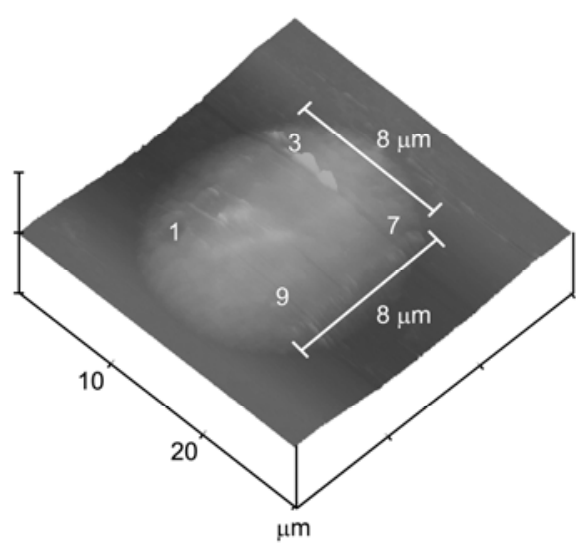

Figure 1 Indentation lattice. 
Table 1 Calculated contact pressures and cutting depths under different vertical loading forces

\begin{tabular}{|c|c|c|c|c|c|}
\hline \multirow{2}{*}{$\begin{array}{l}\text { Loading voltage } \\
\qquad V(\mathrm{~V})\end{array}$} & \multirow{2}{*}{$\begin{array}{l}\text { Vertical loading } \\
\text { force } W(\mu \mathrm{N})\end{array}$} & \multicolumn{2}{|c|}{ Contact pressure $P_{\max }(\mathrm{Pa})$} & \multicolumn{2}{|c|}{ Cutting depth $\delta(\mathrm{nm})$} \\
\hline & & Ovarian cancer cells & Colon cancer cells & Ovarian cancer cells & Colon cancer cells \\
\hline 3.0 & 17.523 & 0.597 & 0.525 & 4.228 & 1.52 \\
\hline 3.5 & 20.444 & 0.538 & 0.475 & 4.686 & 1.69 \\
\hline 4.0 & 23.364 & 0.493 & 0.434 & 5.122 & 1.84 \\
\hline 4.5 & 26.285 & 0.455 & 0.398 & 5.540 & 2.00 \\
\hline 5.0 & 29.206 & 0.424 & 0.373 & 5.944 & 2.14 \\
\hline 5.5 & 32.126 & 0.398 & 0.350 & 6.334 & 2.28 \\
\hline
\end{tabular}

tween different cell types, calculated for the same loading voltage. This suggests that the elastic modulus differed considerably between the different cell types.

\subsection{Measured cutting depths under different loading forces}

The topographies of the ovarian cells and colon cancer cells after each cutting process are shown in Figures 2 and 3, respectively. The depth of the square cutting area on the cell surface increased with increased loading voltage. Because the cell organelles and cytoskeleton are distributed stochastically in the cells [12-14], the surface of the cutting area was uneven, and some organelles and cytoskeleton were exposed; some of the exposed organelles were complete, while others were cut. As shown in Figure 2, four pits of different sizes were seen, probably where organelles had been lost.

The topography of the cells and the average cutting depth on the cell surface were collated and analyzed under differ- ent loading voltages. The measurements were then compared with the Hertzian theory calculation, as shown in Figure 4.

As shown in Figure 4, the measured values were in good agreement with the calculations based on Hertzian theory, thus confirming the validity of the theoretical calculations. Comparison of the average elastic modulus of the cell surface and the average intracellular elastic modulus after each cutting process is shown in Figure 5. Based on measurements from 20 cells for each sample, $t$-tests were used to estimate the elastic modulus range of the cells. The average differences between the surface elastic modulus and average elastic modulus for ovarian and the colon cancer cells were $0.288 \pm 0.08 \mathrm{kPa}$ and $0.376 \pm 0.16 \mathrm{kPa}$, respectively. The average intracellular elastic modulus was greater than the average surface elastic modulus, indicating that the elastic moduli of the organelles and cytoskeleton were greater than that of the rest of the cell. It is therefore reasonable to use the average intracellular elastic modulus as indicative of the elastic modulus of the whole cell.
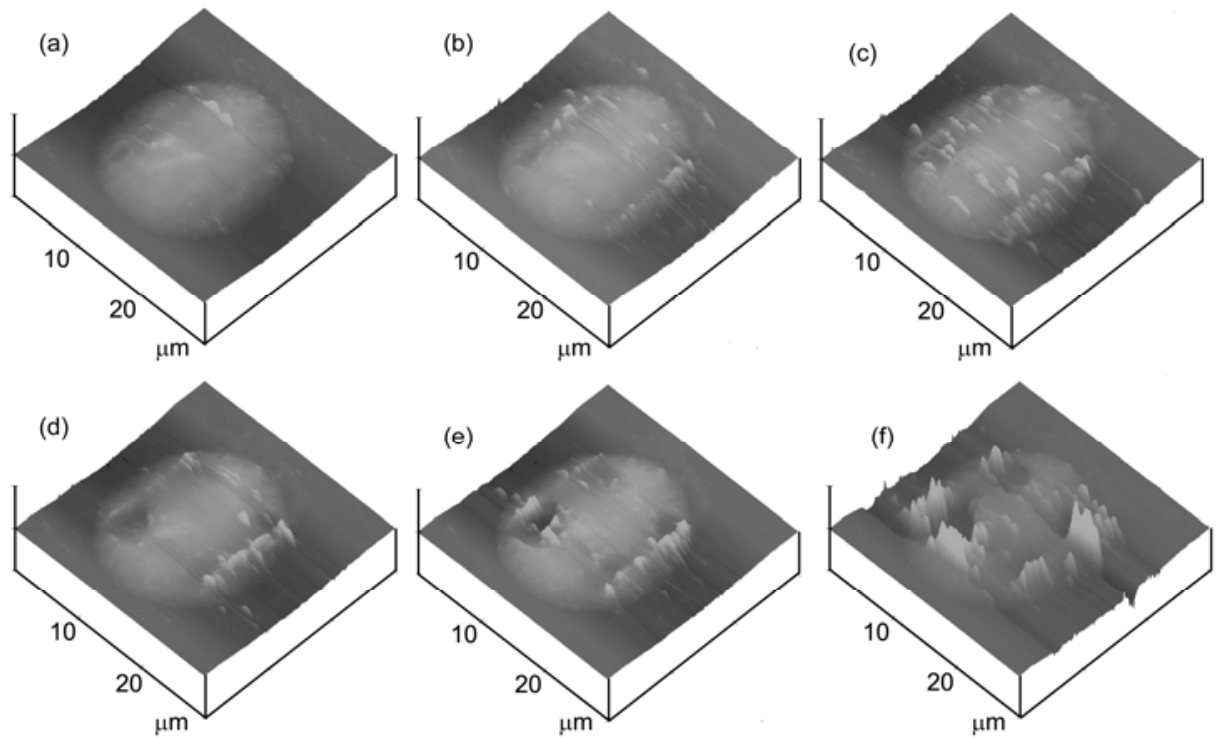

Figure 2 AFM topography of an ovarian cell after each cutting process. (a)-(f) are machined topography under the loading voltage of 3, 3.5, 4, 4.5, 5 and $5.5 \mathrm{~V}$, respectively. 

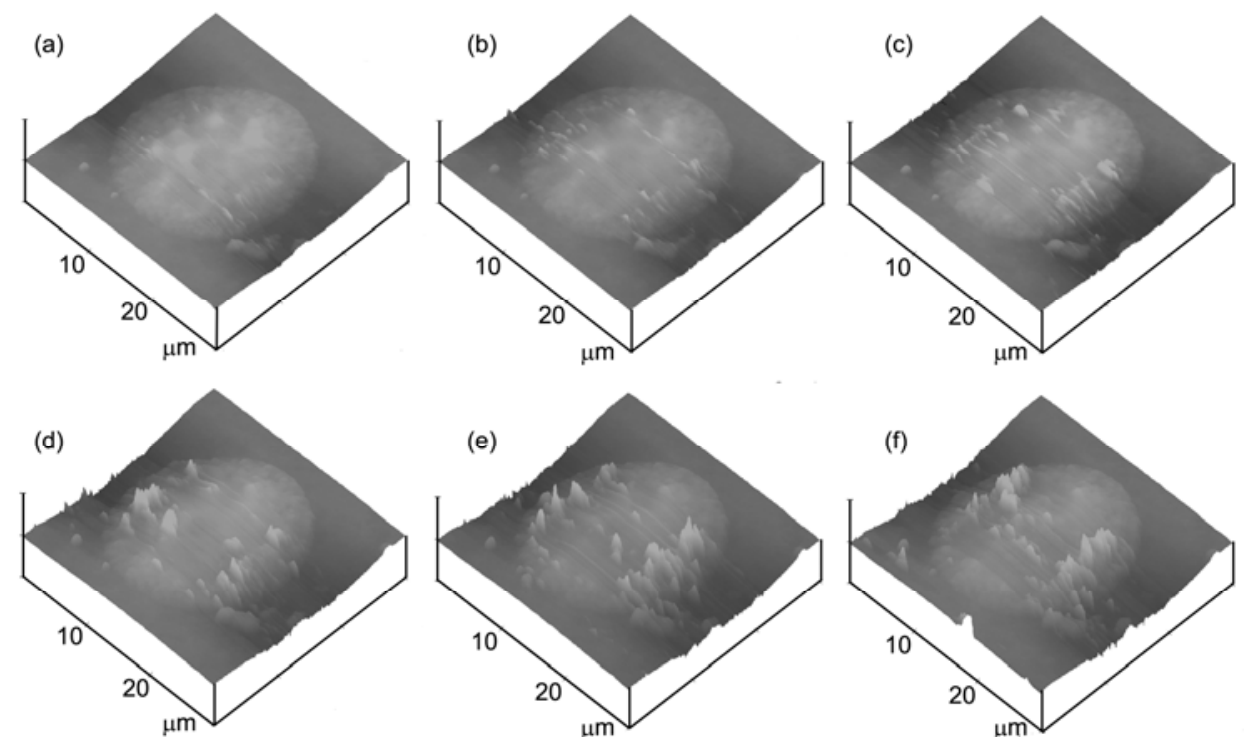

Figure 3 AFM topography of a colon cancer cell after each cutting process. (a)-(f) are machined topography under the loading voltage of $3,3.5,4,4.5,5$ and $5.5 \mathrm{~V}$, respectively.
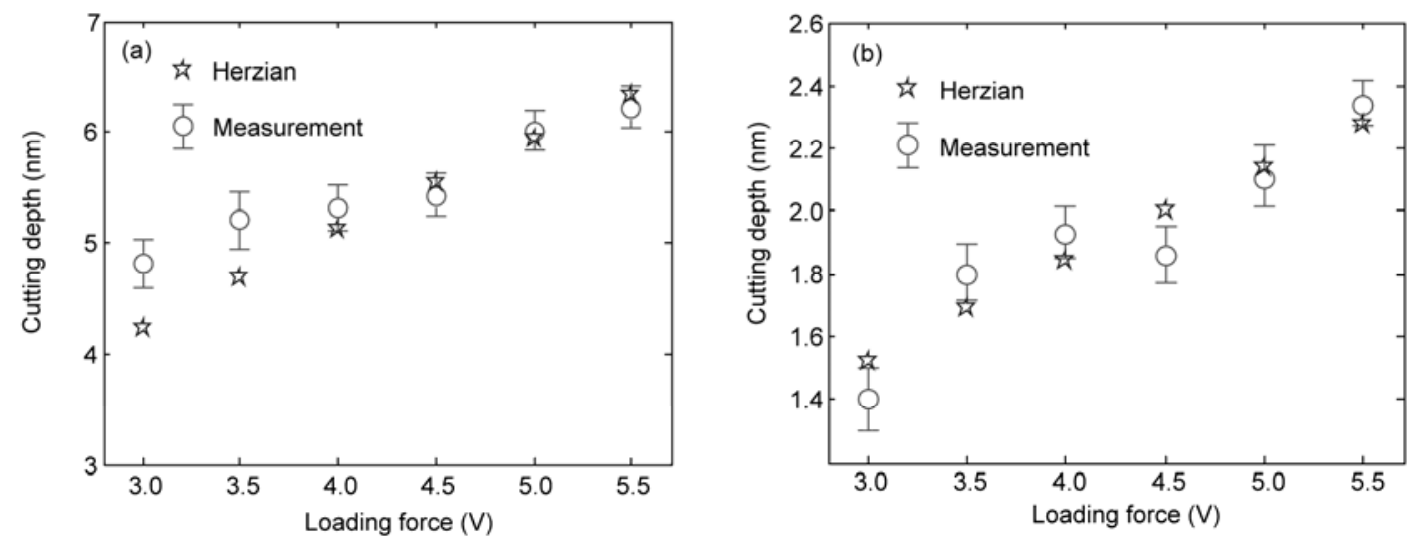

Figure 4 Cutting depth on the cell surface under different loading voltages. Comparison of measurements and Hertzian theory calculations in ovarian cells (a) and colon cancer cells (b).
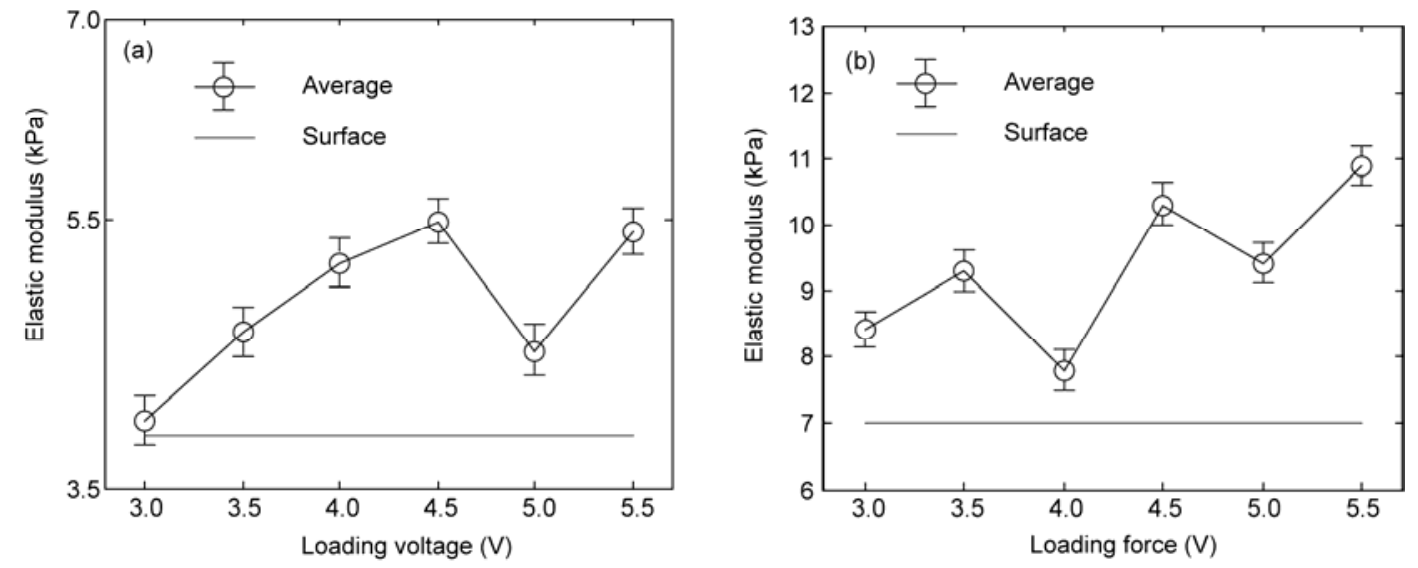

Figure 5 Comparison between the surface elastic modulus and the average elastic modulus in ovarian cells (a) and colon cancer cells (b). 


\section{Discussion}

The intracellular elastic modulus can be measured by cutting cells into sequential layers, allowing accurate comparisons of the elastic modulus to be made among different cell types. Errors caused by inappropriate selection of measurement points are excluded using this technique.

This method may therefore provide the basis for the development of more accurate and reliable methods for detecting and treating cancers. Further studies are needed to observe the cut surface of cells using other optical instruments.

\section{Conclusion}

The surface and internal mechanical properties of cancer cells can be measured using AFM. This study used a new method of measuring the intracellular elastic modulus based on cutting the cells into sequential layers using AFM. Our calculations confirmed that Hertzian theory can also be used to calculate the mechanical properties in the AFM process. The results demonstrated a large difference between the surface elastic modulus and the average intracellular elastic modulus, mainly attributable to the complex structures present within the cell. This method of measuring intracellular mechanical properties may help in the development of more accurate methods of detecting cancers.

This work was supported by the National Natural Science Foundation of China (51175124) and the Self-Planned Task of State Key Laboratory of Robotics and System of Harbin Institute of Technology (SKLRS 200903C).
1 Cai X F, Xing X B, Cai J Y, et al. Connection between biomechanics and cytoskeleton structure of lymphocyte and Jurkat cells: An AFM study. Micro, 2010, 41: 257-262

2 Xie W L, Yang P H, Zeng X, et al. Visual characterization of targeted effect of holo-transferrin-tagged dihydroartemisinin on human breast cancer cells. Chin Sci Bull, 2010, 22: 2390-2395

3 Suresh S. Biomechanics and biophysics of cancer cells. Acta Biomater, 2007, 3: 413-438

4 Smith B A, Tolloczko B, Martin J G, et al. Probing the viscoelastic behavior of cultured airway smooth muscle cells with qtomic force microscopy. Biophys J, 2005, 88: 2994-3007

5 Cross S E, Jin Y S, Rao J, et al. Nanomechanical analysis of cells from cancer patients. Nat Nanotechnol, 2007, 2: 780-783

6 Koinkar V N, Bhushan B. Scaning and transmission electron microscopies of single-crystal silicon microworn/machined using atomic force microscopy. J Mater Res, 1997, 12: 3219-3224

7 Yin Q, Zhuang D M, Jiang Y Q, et al. Establishment of a highthroughput screening system foruniversal anti-HIV targets. Chin Sci Bull, 2010, 55: 937-942

8 Alcaraz J, Buscemi L, Grabulosa M, et al. Microrheology of human lung epithelial cells measured by atomic force microscopy. Biophys $\mathrm{J}$, 2003, 84: 2071-2079

9 Zhang L P, Chen M, Yang P. Effects of ytterbium on outward $\mathrm{K}^{+}$ currents in NIH3T3 cell. Chin Sci Bull, 2010, 55: 246-250

10 Lau A W C, Hoffman B D, Davies A, et al. Microrheology, stress fluctuations, and active behavior of living cells. Phys Rev Lett, 2003, 91: 198101-1-198101-4

11 Hu M Q, Wang J K, Zhao H X, et al. Nanostructure and nanomechanics analysis of lymphocyte using AFM: From resting, activated to apoptosis. J Biomech, 2009, 42: 1513-1519

12 Afrin R, Yamada T, Ikai A. Analysis of force curves obtained on the live cell membrane using chemically modified AFM probes. Ultramicroscopy, 2005, 103: 253

13 Chen P P, Dong H T, Chen L, et al. Application of atomic force microscopy to living samples from cells to fresh tissues. Chin Sci Bull, 2009, 54: 2410-2415

14 Bremmell K E, Evans A, Prestidge C A. Deformation and nano- rheology of red blood cells: An AFM investigation. Colloid Surface B, 2006, 50: 43-48

Open Access This article is distributed under the terms of the Creative Commons Attribution License which permits any use, distribution, and reproduction in any medium, provided the original author(s) and source are credited. 